\title{
Exploring the vertical age structure of the Galactic disc
}

\author{
Luca Casagrande \\ Research School of Astronomy \& Astrophysics, Mount Stromlo Observatory \\ The Australian National University, ACT 2611, Australia \\ email: luca.casagrande@anu.edu.au
}

\begin{abstract}
While in external or high-redshift galaxies we can only measure integrated stellar properties at best, the Milky Way offers us the unique opportunity to study its individual baryonic components, including stars. We use oscillations measured in red giant stars by the Kepler satellite to derive stellar ages and explore the vertical age structure across few $\mathrm{kpc}$ of the Milky Way disc. We find that old stars dominate at increasing Galactic heights, whereas closer to the plane a rich zoology of ages exists. The age distribution of stars shows a smooth distribution over the last $10 \mathrm{Gyr}$, which together with a flat age-metallicity relation is consistent with a quiescent evolution for the Milky Way disc since a redshift of about two.
\end{abstract}

Keywords. Galaxy: stellar content, Galaxy: disc, Galaxy: evolution, stars: fundamental parameters, stars: oscillations, surveys, techniques: photometric

\section{Whole shebang}

Stellar ages are the keystone in setting the time line of the events which shaped the Milky Way disc through its 13 billion year old history. So far "Galactic archaeology" studies have been severely limited by unsuccessful attempts to determine precise ages for large samples of distant stars. This impasse can now be broken thanks to asteroseismology, a technique which allows accurate determination of physical properties of stars, including their ages (e.g., Chaplin \& Miglio 2013).

We use the oscillations measured in 989 red giants by the Kepler satellite to explore the vertical age structure of the Galactic disc across 1,000 parsecs (Casagrande et al. 2014). We find that old stars dominate at increasing Galactic heights, whereas stars closer to the plane exhibit a wide range of ages. This results into a vertical age gradient of approximately $4 \mathrm{Gyr} / \mathrm{kpc}$, though characterized by a large dispersion of ages at all heights. The smooth age distribution of red giants over the last $10 \mathrm{Gyr}$, together with their flat age-metallicity relation indicate a mostly quiescent evolution for the Milky Way disc since $z \simeq 2$ (Casagrande et al. 2015). To interpret this in the context of how Milky-Way like galaxies assemble see e.g. van Dokkum et al. (2013). While increasingly sophisticated cosmological simulations are now able to predict also gross morphological properties on galactic scales, our measurements highlight the importance of internal dynamical processes in disc evolution scenarios, and provide a timescale for them.

\section{References}

Casagrande, L., Silva Aguirre, V., Stello, D., et al. 2014, ApJ, 787, 110

Casagrande, L., Silva Aguirre V., Schlesinger K. J., et al. 2015, MNRAS, in press.

Chaplin, W. J. \& Miglio, A. 2013, ARA\&A, 51, 353

van Dokkum, P. G., Leja, J., Nelson, E. J., et al. 2013, ApJ, 771, L35 